\title{
Dumbbell Bars and Spirals: Structures of Interacting Galaxies (with a Video-Movie)
}

\author{
J. Anosova \\ University of Texas at Austin, Dept. of Aerospace and Engineering, \\ Austin, TX 78712, USA \\ K. Tanikawa \\ National Astronomical Observatory, Tokyo 181, Japan
}

\begin{abstract}
We study the dynamics of spherical systems which consist of extended shells of relatively low-mass particles around and inside the orbit of two heavy centers of gravity (a binary with point-mass components) by computer simulations.
\end{abstract}

\section{Introduction}

We study the dynamical evolution of models of galaxies which contain two heavy centers of gravity and extended shells inside and around orbits of these binaries. The binary components are surrounded by $\mathrm{N}=16000$ small-mass particles in uniform random distribution on a few spherical envelopes with different radii, expanding with some velocity. Some shells are inside the orbit of the binary.

We study the dynamics of these models by computer simulations in the frame of the three-body problem and compilation of numerous initial conditions. It is shown that the shell expands isotropically with decreasing velocity and then falls back into the system forming zones of compressed matter. At some moment of time there could be a collapse of these particles on to the heavier component of the binary. Further in time, some part of particles which were outside the binary orbit escape from the system. Other particles which were initially inside of the orbit are captured by binary components, forming in the center an extended body with a form like a "dumbbell" bar.

For models with smaller radii of shells, about one-half of the particles escape from the systems, while for larger values the shell disrupts as a whole. Most of the escaping particles form collimated flows-spirals in the plane of the orbit of the binary. The form, position and direction of the flows depend on the position of the heavier component of the binary at the moment of the closest approach of the particles and on the ratio of masses of the binary components. Remaining escaping particles form a rather uniform outward "flow". This means that escape of these particles occurs randomly in time and in direction with respect to the center of gravity of a system.

The expanding structure of the particle system depends sensitively on initial conditions. This is because the expansion is due to the gravitational slingshot effect and this effect is sensitive to initial conditions. During the evolution of our 
models, the different kinds of structures seen in the systems often are very similar to the observed structures of galaxies: spiral and elliptical galaxies, interacting galaxies, different kinds of flows, and jets. The systems are totally expanding - after 40 periods of rotation of the binary the system expands by about 300 times.

Our video-movie shows the dynamical evolution of structures in the models with time and sometimes these model structures are similar to observed ones. The formation of different kinds of structures of galaxies depends on the motions of particles with respect to the apsidal line of the components nuclei:

- in the case of almost orthogonal motion (the gravitational slingshot effect), these particles escape from the systems and before an escape form open expanding spirals;

- in the case of small angles of motion of particles with the respect to this line, these particles are captured by the binary components and form double bars in the centers of clusters;

- in the intermediate cases, particles form different kinds of flows like jets, rings, close spirals, etc.

Acknowledgments. The authors thank Alar Toomre for very useful and interesting discussions as well as S. J. Aarseth for kindly sending his TRIPLE code. 